\section{Gliocyte and synapse analyses in cerebral ganglia of the Chinese mitten crab, Eriocheir sinensis: ultrastructural study}

H. Zhang, P. Yu, S. Zhong, T. Ge, S. Peng,

Z. Zhou, X. Guo

College of Animal Science and

Technology, Jiangxi Agricultural

University, Nanchang, China

\begin{abstract}
The Chinese mitten crab Eriocheir sinensis is an economically important aquatic species in China. Many studies on gene structure, breeding, and diseases of the crab have been reported. However, knowledge about the organization of the nerve system of the crab remains largely unknown. To study the ultrastructure of the cerebral ganglia of $E$. sinensis and to compare the histological findings regarding the nerve systems of crustaceans, the cerebral ganglia were observed by transmission electron microscopy. The results showed that four types of gliocytes, including type I, II, III, and IV gliocytes were located in the cerebral ganglia. In addition, three types of synapses were present in the cerebral ganglia, including unidirectional synapses, bidirectional synapses, and combined type synapses.
\end{abstract}

\section{Introduction}

The Chinese mitten crab, Eriocheir sinensis (Crustacea, Decapoda, Varunidae), commonly known as the hairy crab, is native to China and has also spread to Europe and America..$^{1-3}$ The crab is an invasive and nonindigenous species that destroys the local ecological balance in Europe and America. ${ }^{2}$ In China, however, the crab has long been served as a nutritious food and is regarded as a delicacy. ${ }^{2,4} \mathrm{~A}$ large number of the crabs are consumed each year in China. Most commercial E. sinensis are farmed for marketing and consumption. Therefore, crab aquaculture is an important economic industry in several provinces of southeast China. Currently, many studies of $E$. sinensis focus on artificial aquaculture techniques, prevention of pathogenic microorganism invasion, and gene identification. ${ }^{5-7}$ The characterization of hematopoietic tissues of the crab have also been reported. ${ }^{8}$ However, ultrastructural knowledge of the organs of $E$. sinensis is still very limited. In this study, the cerebral ganglia ultrastructure of the crab was observed by transmission electron microscopy (TEM) to further understand the neurobiology of this crustacean. Knowledge of the morphological aspects of the nerve system will facilitate studies of comparative histology and evolutionary biology.

\section{Materials and Methods}

\section{Animals}

The $E$. sinensis were harvested from Junshan Lake (116 $32^{\prime} 84^{\prime \prime}$ E, 28 $\left.41^{\prime} 57^{\prime \prime} \mathrm{N}\right)$, in Jinxian County, Nanchang, Jiangxi Province, China, in September 2014. This work was approved by the Ethical Committee for Animal Care and Use of Jiangxi Agricultural University after relevant ethical review according to the National Institutes of Health Guide for the Care and Use of Laboratory Animals.

\section{Transmission electron microscopy}

TEM was conducted according to the methods of Zhang et al. ${ }^{9}$ Briefly, after 12 crabs were anesthetized on ice, small pieces of cerebral ganglia were quickly excised and fixed in $2.5 \%$ glutaraldehyde/phosphate-buffered saline (PBS) at $4^{\circ} \mathrm{C}$ for $24 \mathrm{~h}$, and then washed three times for $5 \mathrm{~min}$ in $0.01 \mathrm{M}$ PBS (pH 7.4). The samples were postfixed in $1 \% \mathrm{OsO}_{4}$ for $2 \mathrm{~h}$, washed three times for $5 \mathrm{~min}$ in $0.01 \mathrm{M} \mathrm{PBS}$, dehydrated in a concentration series of ethanol, and then dehydrated two times in acetone, embedded in Spurr's resin at $37^{\circ} \mathrm{C}$ for $24 \mathrm{~h}$, and then at $60^{\circ} \mathrm{C}$ for $48 \mathrm{~h}$. The specimens were sectioned by using a LKB-V ultramicrotome (LKB, Stockholm, Sweden), and then the sections were stained with $1 \%$ uranyl acetate and Reynold s lead citrate for 20 min. Finally, the ultrathin sections were observed and photographed by a Hitachi H-600 TEM (Tokyo, Japan).

\section{Results}

\section{Three types of synapses in the cerebral ganglia of $E$. sinensis}

The schematic diagrams of the three types of synapses between neuritis of neurons in the cerebral ganglia of $E$. sinensis were drawn based on their ultrastructure (Figure 1). In the neuropil of cerebral ganglia, three types of synapses were observed by TEM according to their ultrastructural characteristics. One type of synapse, known as the unidirectional synapse, contained classic presynaptic structures with synaptic vesicles and mitochondria, postsynaptic structures, and synaptic clefts (Figures 2 and 3). The other type of synapse, the bidirectional synapses, had synaptic vesicles that were both in close proximity to the
Correspondence: Xiaoquan Guo, College of Animal Science and Technology, Jiangxi Agricultural University, 1101 Zhimin Road, Nanchang 330045, China.

Tel. +86.791.83813503 - Fax: +86.791 .83813504 .

E-mail: xqguo20720@aliyun.com

Key words: Chinese mitten crab; cerebral ganglia; ultrastructure; gliocytes; synapses.

Contributions: HZ, concept and design, data acquisition and interpretation, article drafting, manuscript planning and writing; PY, SZ, TG, experimental work and study execution; SP, experimental assistance; ZZ, technical assistance; $\mathrm{XG}$, concept and manuscript editing. H.Z. and P.Y. contributed equally to this work.

Funding: this work was supported by the National Natural Science Foundation of China (Grant No. 31560681), Science and Technology Program of Jiangxi Province (Grant No. 20151BBF60007), Natural Science Foundation (Grant No. 20122BAB214021), Natural Science Foundation of Department of Education of Jiangxi Province (Grant No. GJJ13262) and Jiangxi Young Scientists Target Training Program to Xiaoquan Guo (Grant No. 20122BCB23022).

Conflict of interest: the authors declare no conflict of interest.

Received for publication: 7 April 2016.

Accepted for publication: 1 July 2016.

This work is licensed under a Creative Commons Attribution-NonCommercial 4.0 International License (CC BY-NC 4.0).

(c) Copyright H. Zhang et al., 2016

Licensee PAGEPress, Italy

European Journal of Histochemistry 2016; 60:2655 doi:10.4081/ejh.2016.2655

geminus neurites (Figures 2 and 3). Another type of synapse, the combined type synapse, was comprised of at least one unidirectional synapse and one bidirectional synapse (Figures 2 and 3). The synaptic vesicles can be classified into two basic types, electron-dense core vesicles and small vesicles (Figure 3 ).

\section{Four types of gliocytes in the} cerebral ganglia of $E$. sinensis

The cerebral ganglia of $E$. sinensis consisted of many axons and gliocytes. The axons were separated by elongated cytoplasmic processes of gliocytes, which were electron-dense gliocytes and electron-lucent gliocytes, based on their cytoplasmic electron density (Figure 4). The processes of electron-lucent gliocytes contained some vesicular structures with unit membrane structures (Figure 5). Axons were wrapped by glial membrane that separates neighboring axons from one another (Figures 4 and 5). 
Microtubules were present in the processes of the gliocytes. Four types of gliocytes were present in the cerebral ganglia. Type I gliocytes contained mitochondria, peroxisomes, small vacuoles and abundant rough endoplasmic reticulum in the cytoplasm (Figure 6). In type II gliocytes, the organelles were rare, but many small vacuoles of various sizes were scattered throughout the cytoplasm (Figure 7). Type III gliocytes contained various organelles in the cytoplasm, such as mitochondria, Golgi complexes and peroxisomes (Figure 8). The characteristic feature of type IV gliocytes was the presence of large and electron-dense lysosomes containing lipofuscin in the cytoplasm (Figure 9). Type IV gliocytes were observed adjacent to unidirectional synapses between neurons (Figure 9).

\section{Discussion}

In previous studies, the ultrastructure of the motor nerve terminal, stomatogastric nervous system, nerve fibers of the protocerebral tract, and glial cells of the central nervous system have been investigated in various species of crabs, such as Callinectes sapidus, Cancer

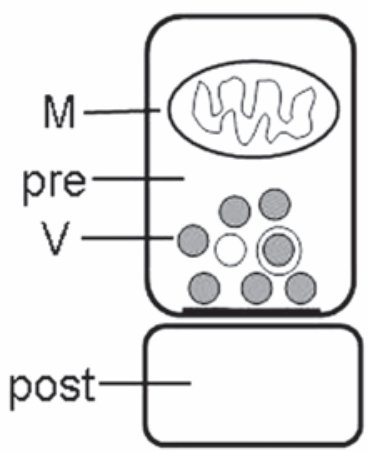

unidirectional synapse

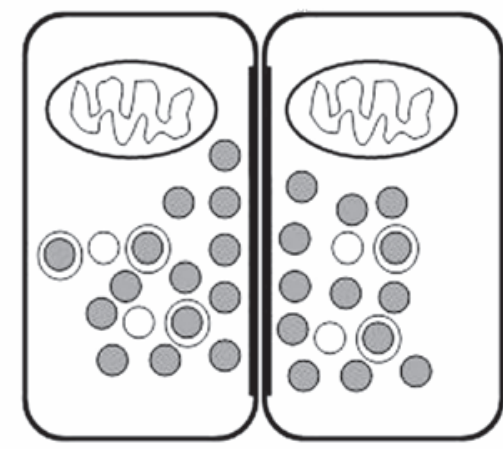

bidirectional synapses

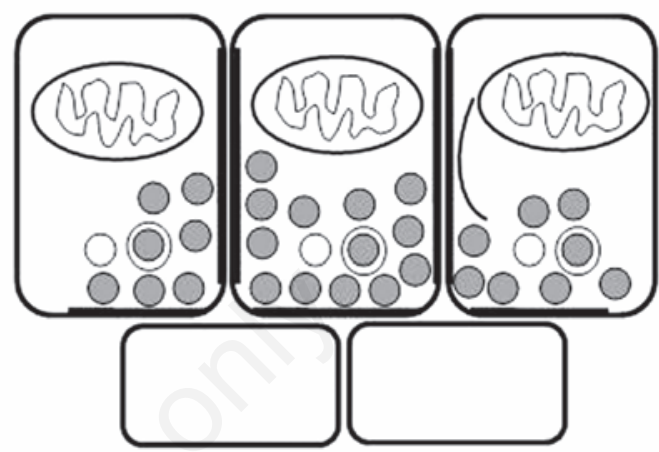

combined type synapses

Figure 1. Schematic diagrams of three types of synapses between neuritis of neurons in the cerebral ganglia of $E$. sinensis. post, postsynaptic profiles; pre, presynaptic profiles; $\mathrm{M}$, mitochondrion; V, synaptic vesicles.

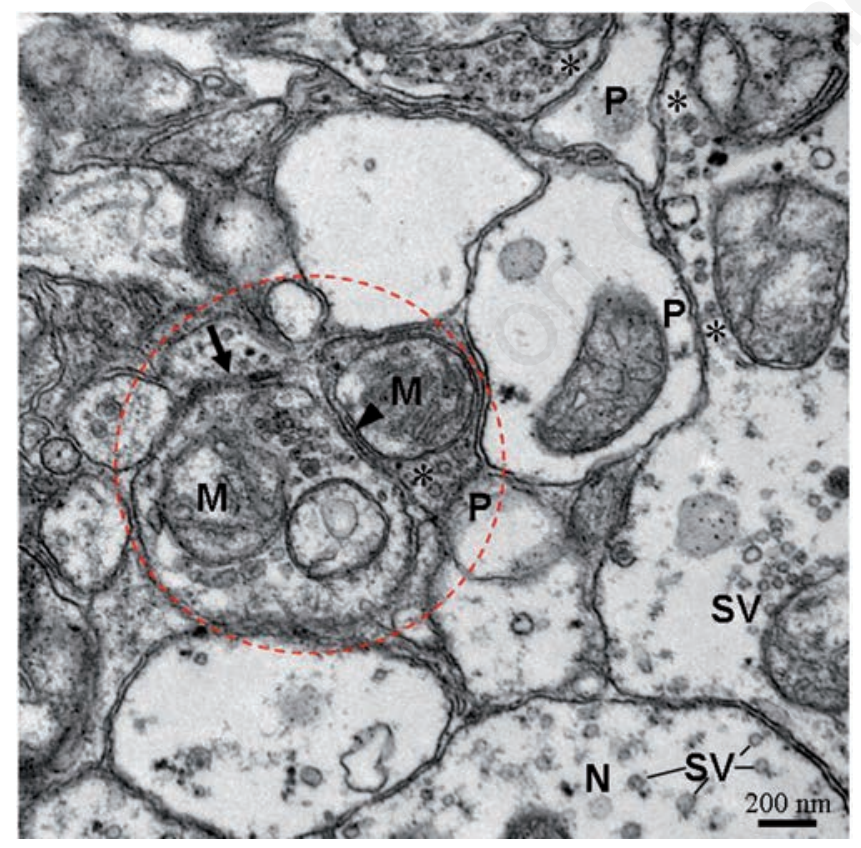

Figure 2. Three types of synapses including unidirectional synapses (asterisk and P), bidirectional synapses (arrows) and combined type synapses (dotted-line circle) in neuropil of cerebral ganglia. The close association of an internal membrane compartment (arrowhead) with the membrane of neurite. P, postsynaptic profiles; asterisk, presynaptic profiles; SV, small vesicles; $\mathrm{N}$, neurite; $M$, mitochondrion.

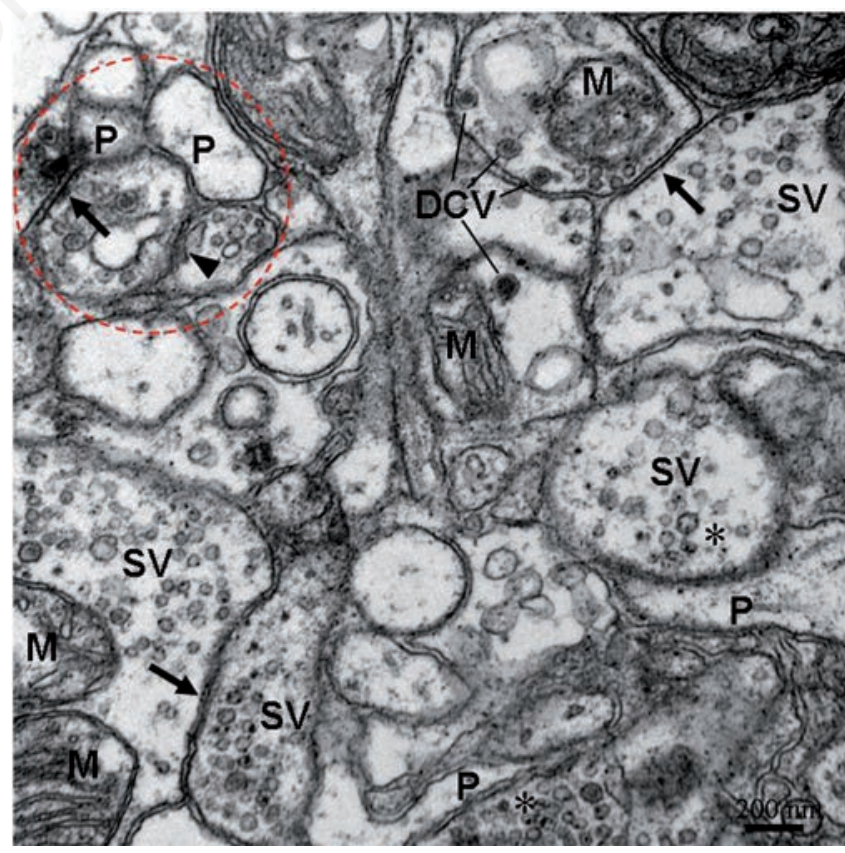

Figure 3. The ultrastructure of neuropil containing three types of synapses. The bidirectional synapses (arrows) with electron-dense core vesicles (DCV)/small vesicles (SV) in both neurites. Dottedline circle lables combined type synapses. The close association of an internal membrane compartment (arrowhead) with the membrane of neurite. $P$, postsynaptic profiles; asterisk, presynaptic profiles; M, mitochondrion. 


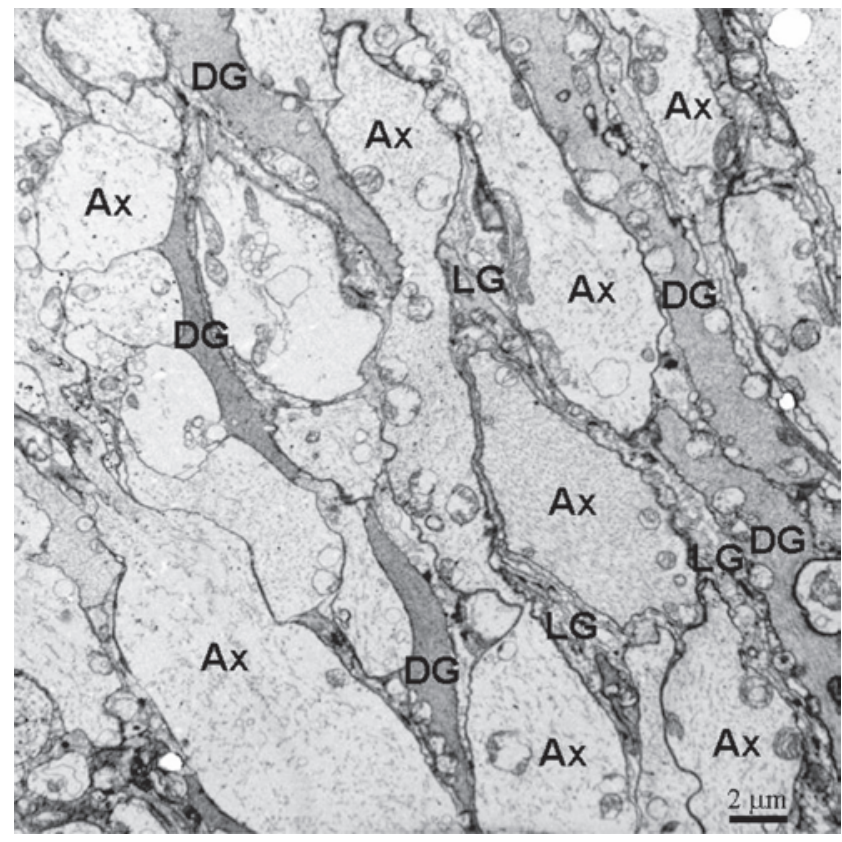

Figure 4. The neuropil in the cerebral ganglia, showing the axons (Ax) are separated by electron-dense gliocytes (DG) and denselucent gliocytes (LG) cytoplasmic processes.

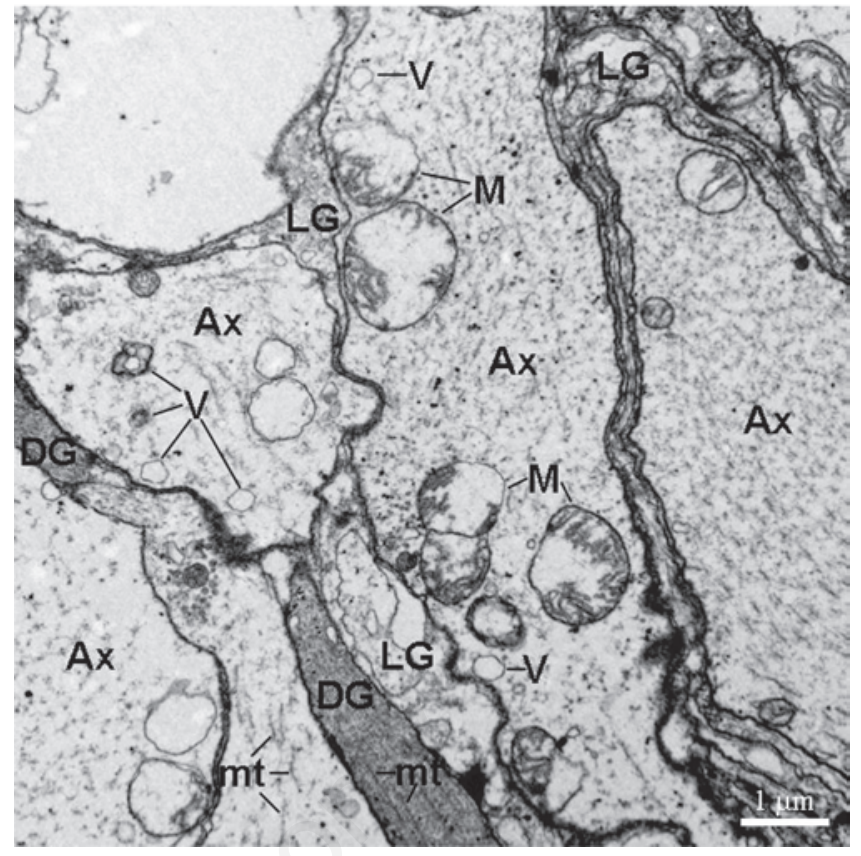

Figure 5. The higher magnification micrograph of neuropil. The glial processes divide axons ( $\mathrm{Ax}$ ) into compartments. The vesicular structures $(\mathrm{V})$ and microtubules (mt) are present in the $\mathbf{A x}$. DG, electron-dense gliocytes; LG, dense-lucent gliocytes; $M$, mitochondrion.

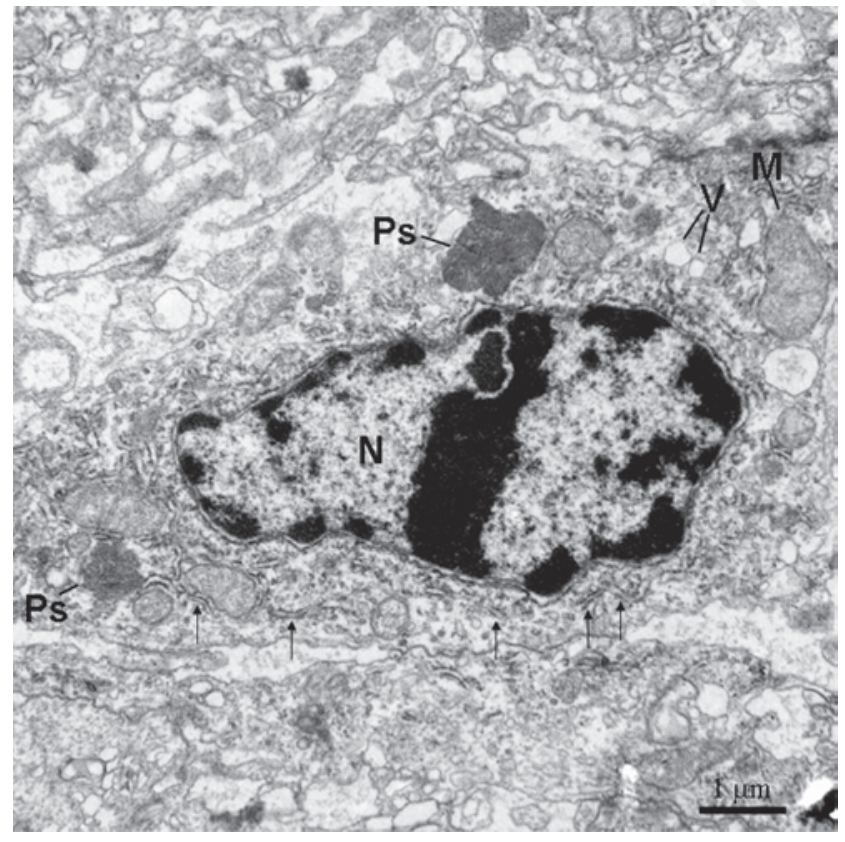

Figure 6. The type I cell contains rich rough endoplasmic reticulum (thin arrows) and two peroxisomes (Ps). N, nucleus; V, vacuoles; $\mathrm{M}$, mitochondrion.

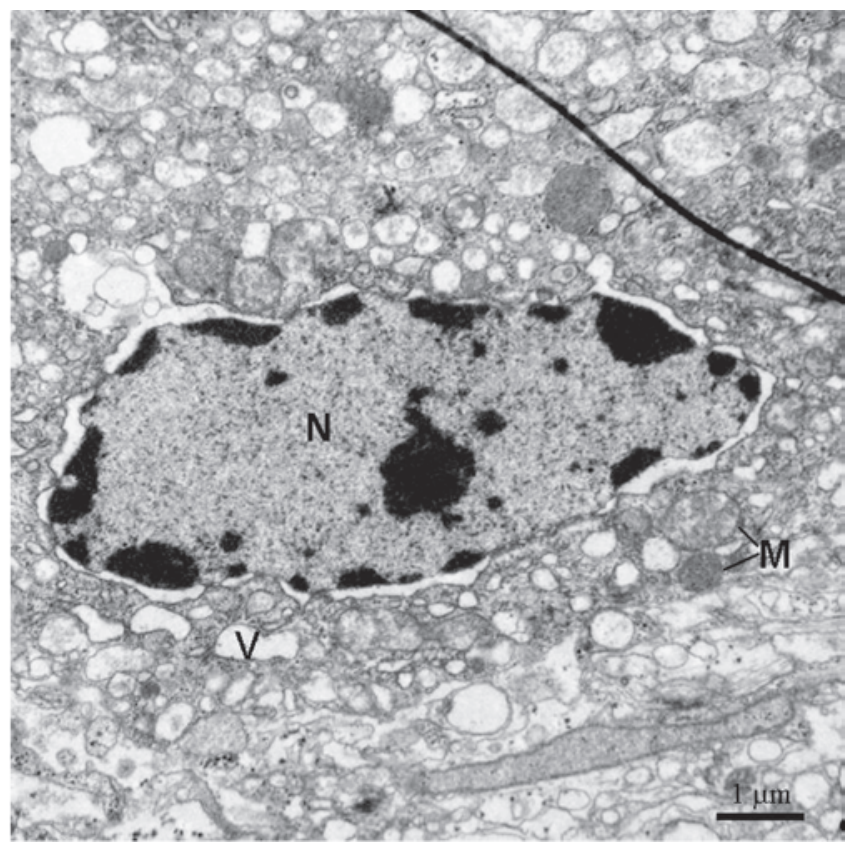

Figure 7. Type II are located in cerebral ganglia. The feature of type II cells is that their cytoplasm containing many vesicles $(\mathrm{V})$. The nucleus is big and containing abundant euchromatin and sparse heterochromatin. Perinuclear gap is visible. M, mitochondrion; $N$, nucleus. 


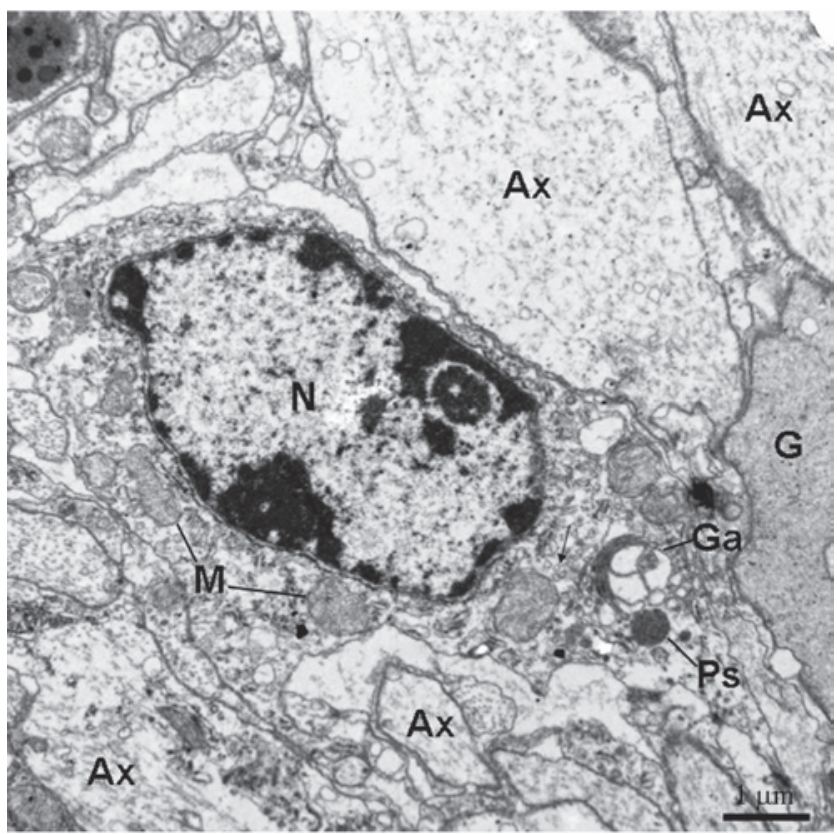

Figure 8. Type III cell is present between axons/glial cytoplasmic processes. The organelles including mitochondria $(\mathrm{M})$ and Golgi apparatus $(\mathrm{Ga})$ are visible. The rough endoplasmic reticulum is rare. Thin arrows show glial tubular lattice clusters. Ax, axon; Ps, peroxisomes; $\mathrm{N}$, nucleus; $\mathrm{G}$, gliocyte.

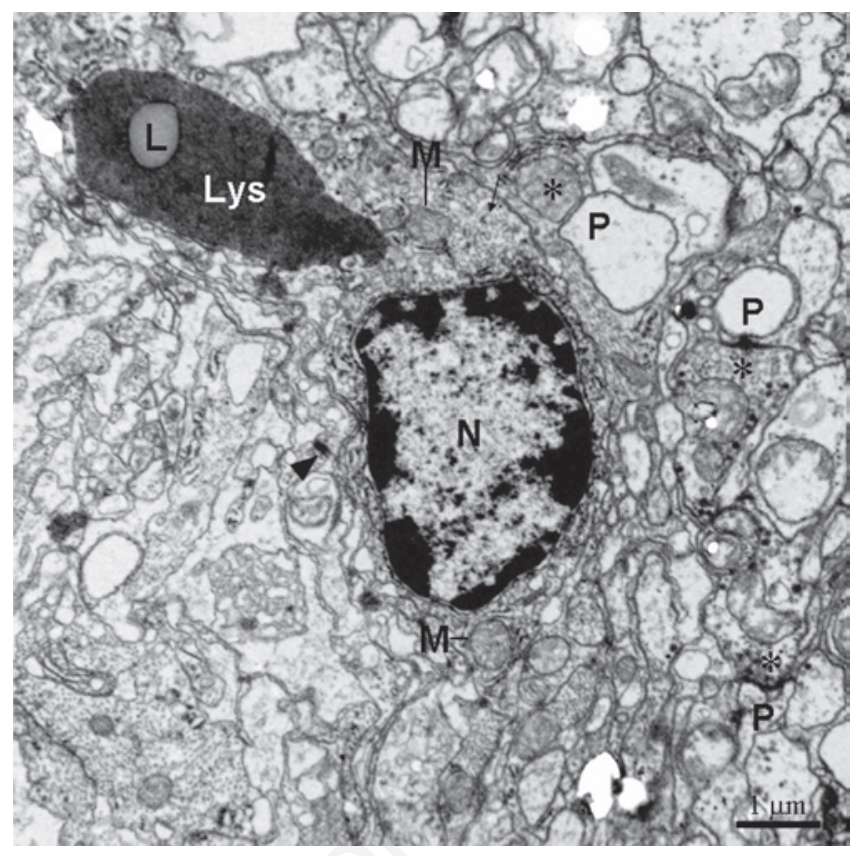

Figure 9. Type IV in cerebral ganglia containing typical large lysosomes (Lys) with lipofuscin (L). Electron-dense heterochromatin is present in edge of the nucleus. Unidirectional synapses are located nearby containing presynaptic profile with small vesicles (asterisks) and postsynaptic profile $(\mathrm{P})$. Asterisks, presynaptic profiles. Thin arrows show glial tubular lattice clusters. Arrowhead, a desmosome join the processes of adjacent cells. M, mitochondrion; $\mathrm{N}$, nucleus. pagurus, and Ucides cordatus. ${ }^{10-14}$ Recently, the neuronal microstructure in the central nervous system of the female mud crab (Scylla olivacea) has also been determined. ${ }^{15}$ However, ultrastructural knowledge of the central nervous system of crabs is still rare. In crayfish, another crustacean, a study documented three cell types present in the crayfish brain. ${ }^{16}$ Moreover, a fourth cell type was identified in another study. ${ }^{17}$ In the present study, the ultrastructure of the cerebral ganglia of $E$. sinensis was observed by TEM. Four types of gliocytes were found in the cerebral ganglia, according to their ultrastructural characteristics. The results suggested that four types of gliocytes are present in the brain of the crustacean. Type I cells contained various organelles in the cytoplasm, such as the rough endoplasmic reticulum, mitochondria, peroxisomes, and endoplasmic reticulum. The presence of these structures suggests that type I cells are active and can play various roles. Rich rough endoplasmic reticulum suggests they can produce a large number of proteins. Generally, peroxisomes contain various oxidases and hydrogen peroxidases, including catalase, xanthine oxidase and urate oxidase. ${ }^{18}$ Peroxisomes can carry out various oxidative reactions that are tightly regulated to adapt to the changing needs of type I cells and to varying external environmental factors. ${ }^{19}$ Therefore, type I cells might be a responding cells. They might play an important role in oxygen use, fatty acid and lipoprotein metabolism, and toxic substance deactivation. Abundant vesicles were present in the cytoplasm of type II cells. It is suggested that type II cells might play an autophagy role in the cerebral ganglia. Autophagy by vesicles plays a critical role in cell metabolism by degrading and recycling internal components when challenged with limited nutrients. ${ }^{20}$ The type III cells could play a role in protein synthesis and processing based on the presence of mitochondria and the Golgi apparatus. The type IV cells could possess phagocytic activity because the lysosomes are present in the cytoplasm, similar to what is found in microglia. Additionally, glial tubular lattice were found in the type III and IV cells. In ventral nerve cord of the crayfish (Procambarus clarkii), the glial tubular lattice was also observed. The tubular lattice plays an active role in adaxonal space $\mathrm{K}^{+}$homeostasis. ${ }^{21}$

In the present study, three types of synapses, unidirectional, bidirectional, and combined type synapses, were also observed in the cerebral ganglia according to their ultrastructural characteristics. The unidirectional and bidirectional synapses (also referred to as asymmetric and symmetric synapses, respectively) are also present in the nervous system of some lower animals, for example, in the ring nerve of the box jellyfish (Tripedalia cystophora) and in the central nervous system of Symsagittifera roscoffensis. ${ }^{22,23}$ However, the combined type synapses are not observed in nerves of these animals. In Drosophila, the complex synapses (containing two or more presynaptic profiles) also exist in the nerve terminals. ${ }^{24}$ In this study, the dense projections (also referred to as T-bars, presynaptic density bars, or dense bars) were not observed in the presynaptic membranes of the synapses. The differences in the structures of dense projections imply a diversification of the central structure/function theme in various niches. ${ }^{25}$ Thus, the presynaptic membranes of four types of synapses are not well developed in the active zones. These finding differ from the findings of peripheral synapses of other arachnids and crustaceans..$^{26-28}$ Herein, however, an internal membrane compartment (also referred to as the subsynaptic cistern) was located adjacent to the inside of the axolemma. The membrane compartment is also present in myelin of the copepod (Bestiolina similis), in brain neurons of Drosophila, and in Onychophoran cephalic ganglia. ${ }^{29-31}$ They are thought to function as smooth endoplasmic reticulum, and could play a role in responses to $\mathrm{Ca}^{2+}$ influx, synthesis of transmembrane proteins, and neurotransmitter release during neuronal communication. ${ }^{32}$ 


\section{References}

1. Herborg LM, Rushton SP, Clare AS, Bentley M. Spread of the Chinese mitten crab (Eriocheir sinensis H. Milne Edwards) in Continental Europe: analysis of a histori cal data set. Hydrobiologia 2003;503:21-8.

2. Clark PF, Mortimer DN, Law RJ, Averns JM, Cohen BA, Wood D, et al. Dioxin and PCB contamination in Chinese mitten crabs: human consumption as a control mechanism for an invasive species. Environ Sci Technol 2009;43:1624-9.

3. Wu JL, Kang XJ, Guo MS, Mu SM, Zhang $\mathrm{ZH}$. Cloning and Functional Analysis of Histones H3 and H4 in Nuclear Shaping during Spermatogenesis of the Chinese Mitten Crab, Eriocheir sinensis. PLoS One 2015;10:e0126623.

4. Chen DW, Zhang M, Shrestha S. Compositional characteristics and nutritional quality of Chinese mitten crab (Eriocheir sinensis). Food Chem 2007;103:1343-9.

5. Ding Z, Meng Q, Liu H, Yuan S, Zhang F, Sun M, et al. First case of hepatopancreatic necrosis disease in pond-reared Chinese mitten crab, Eriocheir sinensis, associated with microsporidian. J Fish Dis 2016 doi: 10.1111/jfd.12437.

6. Song L, Bian C, Luo Y, Wang L, You X, Li J, et al. Draft genome of the Chinese mitten crab, Eriocheir sinensis. Gigascience 2016;5:5.

7. Zhuang K, Wu N, Wang X, Wu X, Wang S, Long $X$, et al. Effects of 3 feeding modes on the volatile and nonvolatile compounds in the edible tissues of female Chinese mitten crab (Eriocheir sinensis). J Food Sci 2016;81:S968-81.

8. Jia Z, Kavungal S, Jiang S, Zhao D, Sun M, Wang L, et al. The characterization of hematopoietic tissue in adult Chinese mitten crab Eriocheir sinensis. Dev Comp Immunol 2016;60:12-22.

9. Zhang H, Guo X, Zhong S, Ge T, Peng S, Yu $P$, et al. Heterogeneous vesicles in mucous epithelial cells of posterior esophagus of Chinese giant salamander (Andrias davidianus). Eur J Histochem 2015;59:
2521.

10. Allodi S, Taffarel M. Electron microscopy of glial cells of the central nervous system in the crab Ucides cordatus. Braz J Med Biol Res 1999;32:327-31.

11. Skiebe P, Ganeshina O. Synaptic neuropil in nerves of the crustacean stomatogastric nervous system: an immunocytochemical and electron microscopical study. J Comp Neurol 2000;420:373-97.

12. Govind CK, Hirji R. Free-floating active zone dense bar in a crab motor nerve terminal. Synapse 2002;43:145-9.

13. Honsa KJ, Govind CK. Structural definition of the neuromuscular system in the swimming-paddle opener muscle of blue crabs. Cell Tissue Res 2002;307:411-21.

14. Corrêa CL, Allodi S, Martinez AM. Ultrastructural study of normal and degenerating nerve fibers in the protocerebral tract of the crab Ucides cordatus. Brain Behav Evol 2005;66:145-57.

15. Kornthong N, Tinikul Y, Khornchatri K, Saeton J, Magerd S, Suwansa-Ard S, et al. Neuronal classification and distribution in the central nervous system of the female mud crab, Scylla olivacea. Microsc Res Tech 2014;77:189-200.

16. Zhang Y, Allodi S, Sandeman DC, Beltz BS. Adult neurogenesis in the crayfish brain: proliferation, migration, and possible origin of precursor cells. Dev Neurobiol 2009;69:415-36.

17. da Silva PG, Benton JL, Beltz BS, Allodi S. Adult neurogenesis: ultrastructure of a neurogenic niche and neurovascular relationships. PLoS One 2012;7:e39267.

18. Angermüller S, Islinger $M$, Völkl A. Peroxisomes and reactive oxygen species, a lasting challenge. Histochem Cell Biol 2009;131:459-63.

19. Smith JJ, Aitchison JD. Peroxisomes take shape. Nat Rev Mol Cell Bio 2013;14:803817.

20. Gallagher LE, Williamson LE, Chan EY. Advances in autophagy regulatory mechanisms. Cells 2016;5:24.

21. Beshay JE, Hahn P, Beshay VE, Hargittai PT, Lieberman EM. Activity-dependent change in morphology of the glial tubular lattice of the crayfish medial giant nerve fiber. Glia 2005;51:121-31.
22. Garm A, Poussart Y, Parkefelt L, Ekström P, Nilsson DE. The ring nerve of the box jellyfish Tripedalia cystophora. Cell Tissue Res 2007;329:147-57.

23. Bery A, Cardona A, Martinez P, Hartenstein V. Structure of the central nervous system of a juvenile acoel, Symsagittifera roscoffensis. Dev Genes Evol 2010;220:61-76.

24. Shayan AJ, Atwood HL. Synaptic ultrastructure in nerve terminals of Drosophila larvae overexpressing the learning gene dunce. J Neurobiol 2000;43:89-97.

25. Bruckner JJ, Zhan H, O'Connor-Giles KM. Advances in imaging ultrastructure yield new insights into presynaptic biology. Front Cell Neurosci 2015;9:196.

26. Coulthard R, Govind CK. Regeneration of phasic synapses on a crayfish slow muscle following allotransplantation of a mixed phasic-tonicnerve. J Neurocytol 2001;30: 231-41.

27. Fabian-Fine R, Seyfarth EA, Meinertzhagen IA. Peripheral synaptic contacts at mechanoreceptors in arachnids and crustaceans: morphological and immunocytochemical characteristics. Microsc Res Tec 2002;58:283-98.

28. Hamilton J, Dillaman RM, Worden MK. Neuromuscular synapses on the dactyl opener muscle of the lobster Homarus americanus. Cell Tissue Res 2006;326:823-34.

29. Peña-Contreras Z, Mendoza-Briceño RV, Miranda-Contreras L, Palacios-Prü EL. Synaptic dimorphism in Onychophoran cephalic ganglia. Rev Biol Trop 2007;55: 261-7.

30. Oh HW, Campusano JM, Hilgenberg LG, Sun X, Smith MA, O'Dowd DK. Ultrastructural analysis of chemical synapses and gap junctions between Drosophila brain neurons in culture. Dev Neurobiol 2008;68:281-94.

31. Wilson CH, Hartline DK. Novel organization and development of copepod myelin. ii. Nonglial origin. J Comp Neurol 2011;519:3281-305.

32. Spacek J, Harris KM. Three-dimensional organization of smooth endoplasmic reticulum in hippocampal CA1 dendrites and dendriticspines of the immature and mature rat. J Neurosci 1997;17:190203. 